\title{
1 In-silico interaction of Hydroxychloroquine drug with various proteins of coronavirus 2 (SARS-CoV-2): A Computational approaches to combat COVID-19
}

3 Rishee K. Kalaria ${ }^{1 *}$ and Hiren K. Patel $^{2}$

$4 \quad{ }^{1}$ ASPEE Shakilam Biotechnology Institute, Navsari Agricultural University, Surat (Gujarat)-395007

$5 \quad{ }^{2}$ School of Science, P. P. Savani University, Surat(Gujarat)-394125

6 * Corresponding author: risheekal@nau.in (ORCID-0000-0002-4481-3834)

7 Abstract:

8 Most human viral illnesses are a result of a pathogenic occurrence. Some of the diseases caused by these 9 transmissible events have infected millions of people around the world, with some contributing to elevated morbidity/mortality rates in humans. Changes in the viral proteins that act as host receptor ligands may promote spill over between organisms. Finding a remedy along with the putative mechanism to cure COVID-19 spread is the urgent need of recent time. Even though limited amount of data are available, utilizing In silico approaches can be promising for the action. In the present study, In silico approach were performed using receptor-binding domain of Envelop protein, PLpro protein and Spike glycoprotein of SARS-CoV-2 and its interaction with drug Hydroxychloroquine for hinders the epidemic. Based on available data of SARS-CoV and SARS-CoV-2, target proteins structure were predicted using homology modelling and further structures stabilization check using Ramachandran plot. Identification of pockets and cavities in all potential targets performed using CASTp web server and energy minimization was carried out in order to dock these potential targets with the candidate drug Hydroxychloroquine using Patchdock web server. In silico docking study showed that hydroxychloroquine drug interactions with SARS-CoV2 show a higher binding affinity with spike glycoprotein and PLPRO protein compared to protein envelopes that could be ladder for potential targeting and synthesizing of another aniviral drug. In silico methods used in this study, the efficacy of a wide variety of repositioned and/or novel drug candidates could also be tested prior to clinical evaluation.

Keywords: SARS-CoV2, CASTp, hydroxychloroquine, Molinspiration, ADMET and Patchdock web server

\section{Introduction:}

The world is facing a dire situation of global public health emergency due to a viral pandemic of severe febrile pneumonia like respiratory syndrome caused by novel coronavirus, provisionally named as 2019-nCoV. Coronaviruses (CoVs) cause various diseases in large ranges of vertebrates including humans. Although CoVs were previously only associated with a common cold, new CoVs related to the "severe acute respiratory syndrome" (SARS-CoV) discovered in China's human population for the first time in 2002 caused 10 percent of the total worldwide cases to die [1];[2]. In Wuhan, China was first reported the rapidly spreading, highly contagious and pathogenic SARS-coronavirus 2 (SARS-CoV-2) associated Coronavirus Disease 2019 (COVID19) and also been declared as a pandemic by the World Health Organization (WHO) [3]. Nonetheless, it was initially known as a novel coronavirus, namely 2019-nCoV. The World Health Organization (WHO) later called the virus "Severe Acute Respiratory Syndrome Coronavirus 2" (SARSCoV2) and identified as "Coronavirus Virus 2019" (COVID-19) the disease caused by it. SARS-COV-2, a member of the Coronaviridae family, is a 
type of positive-sense, single-stranded enveloped RNA viruses responsible for causing infections in avian, mammalian and marine species across the world [4]. The 2019-nCov SARS-CoV-2 or Wuhan is identified as the seventh strain of human coronaviruses. Based on phylogenetic analysis and taxonomy, ICTV recognized the novel coronavirus as a sister to severe SARS-CoV and thus designated it as Severe Acute Respiratory Syndrome coronavirus 2 (SARS-CoV-2) [5].

Coronaviruses had the largest genomes of all recognized RNA viruses (26.4-31.7 kb). Assortment of little size ORFs are accessible between the different conserved gene (ORF1ab, spike, envelope, film and nucleocapsid) and, downstream to the nucleocapsid gene in various coronavirus lineages [6]. Approximately 66 per cent of the viral RNA gene, essentially located in the first ORF (ORF1a / b), decipheres two polyproteins, pp1a and pp1ab, encoding 16 non-structural proteins (NSP), while the remaining ORFs encode decoration and basic proteins. The rest some portion of infection genome encodes four crucial auxiliary proteins, including spike (S) glycoprotein, little envelope (E) protein, framework (M) protein, and nucleocapsid (N) protein [7]. It encodes, to be exact, the papain-like protease (PLpro) and the 3-chymotrypsinlike protease (3CLpro), for two major polyproteins which are additionally treated by virally encoded cysteine proteases. Viral polyprotein preparation is central to the production and infectivity of the infection [8]. On account of the significant jobs these two proteases play in the viral life-cycle, they are significant focuses to direct further examinations on the potential helpful targets [9].

At present, there is no clinically shown vaccinations and medicine for COVID-19 neutralization and treatment as per U.S. FDA and WHO. Exploring approved drugs to treat COVID-19 is urgently required. Due to a lack of time the best option is to reconfigure the drug against the COVID-19 goal with various computation approaches. A low-cost antimalarial drug chloroquine and its derivative hydroxychloroquine (HCQ), along with several other antiviral drugs used for combating COVID-19. Hydroxychloroquine sulfate is another form of HCQ which is used for oral administration. HCQ is widely used for Pharmacokinetics because of it readily absorbed in gastrointestinal and easy disposal from renal. However, the clinical signs and poisonous dosages of these medications somewhat vary [10]. In vitro, hydroxychloroquine appears as a versatile bioactive agent reported to possess antiviral activity against RNA viruses as diverse as hepatitis C virus [11] , Zika virus [12], and Ebola virus [13], as well as various DNA viruses such as hepatitis B virus [14]. HCQ has latent therapeutic advantages against SARS-CoV-1 [15]. Hydroxychloroquine has also been reported to inhibit HCoV-229E replication in epithelial cultures of the lung cells in vitro [16]; [17]. Since of its wide range of action against viruses, including most coronaviruses and in particular its close relative SARSCoV-1, the potential effects of hydroxychloroquine on SARS-CoV-2 can be investigated according to preliminary reports [18]. Hydroxychloroquine is probably the first molecule to be used in China and abroad on the front line for the treatment of SARS CoV- 2 infections [19].

In silico studies have played crucial role in candidate drug prediction, computer aided drug design and molecular interaction in past decade [20];[21]. To understand the targeted interaction between drugs and SARSCoV-2 proteins that could bind to receptor domains and help stop the spread of the virus. However, a docking analysis of hydroxychloroquine and its effect on target protein SARS-CoV-2 has yet to be conducted. This study thus provides further insight into the interaction of hydroxychloroquine, a common drug, with the SARS-CoV-2 receptor domain of Envelop protein, PLpro protein and Spike glycoprotein. 


\section{Materials and Methods:}

\subsection{Sequence analysis:}

Some of the targeted protein sequences including SARS-CoV2 Envelop protein sequence (ID:QIV65090.1), PLpro (papain-like protease or ORF1ab polyprotein) protein sequence (ID: QIV65087.1) and Spike glycoprotein sequence (surface glycoprotein) (ID: QIV65088.1) were retrieved from NCBI. Subsequently, all the targeted protein sequence of "SARS-CoV2" were subjected to comparison with available PDB structure using NCBI's tool "BLASTp" [22];[23].

\subsection{Protein Homology Modeling:}

Based on BLASTp result, Protein structure with low resolution and good E-value were selected as templates. All the target protein sequence (Envelop protein, PLpro protein and Spike glycoprotein) along with template crystal structure of SARS-CoV proteins (PDB ID: 5X29_A, 7BTF_A, 6VSB_A) respectively were uploaded to SWISS-MODEL server for automated protein structure homology modelling [24];[25]. Best models were obtained, and each structure was further evaluated.

\subsection{Protein structure validation and energy minimization:}

ProSA is a web-based testing tool focused on a statistical study of all available protein structures used to test the 3D query protein structure model for potential errors. This web tool's performance consists of Z-score, and residue scores map. Z-score measures the consistency of the input protein in the overall model [26];[27]. These models were further subjected to analyze the improvement in energy-minimized protein. Evaluation of built model quality using swiss model was analyze through amino acid region in Ramachandran plot in procheck web server [28]; [21]. The models were chosen based on the percentage of support and frequency of outliers, which could be used for further study. The energy minimization stage ensures that the modeled protein structure remains stable. Using ModRefiner we bserver, the modeled target protein structures were minimized to energy after structure validation. Refinements of the structures were accomplished by performing "main chain energy minimization" and then "strong fullatomic energy minimization" [26].

\subsection{Binding site prediction:}

CASTp server has acknowledged active site of all projected models (Computer Atlas of Surface Topology of protein). CASTp, which mechanically locates and calculates protein pockets and cavities, is based on precise methods of computational geometry as well as alpha form and distinct theory of flows. CASTp identification and capability of open external pockets as well as inaccessible interior cavities by identifying, delineating and measuring hollow external regions on three-dimensional protein structures [29].

\subsection{Ligand Preparation:}

The compound hydroxychloroquine as ligand in smiles structure was downloaded from PubChem database. The PubChem obtained structures are then translated to PDB files using the Free Babel converter tool. 

as a Swiss-knife chemoinformatics [30].

\subsection{Screening of compounds:}

\subsubsection{Evaluation of Lipinski parameters for drug-likeness:}

A drug likeliness property of hydroxychloroquine compound was analysed using Molinspiration tool. For that, input was given in the form of smiles of compound. Molecular properties and bioactivity of drugs with strong affinity prediction using Molinspiration server [31].

\subsubsection{ADMET analysis:}

For ADMET study, ligand saved in smiles format has been uploaded to SwissADME, PROTOX-II and admetSAR webservers. SwissADME is a software resource for predicting ADME and molecular pharmacokinetic properties. The expected outcome consists of lipophilicity, water solubility, physicochemical properties, pharmacokinetics, pharmaco-like and medicinal chemistry [32]. PROTOX-II is a rodent oral toxicity server predicting LD50 value and toxicity class of query molecule into six different classes ranging LD5-5000 with their consequences [33]. AdmetSAR provides ADMET profiles for query molecules and can predict approx 50 ADMET properties including toxicity classes [34];[35]. Ligand was further subjected to remove the Pan Assay Interference Compounds (PAINS) and for its exclusion in bioassays [36].

\subsection{In silico docking:} patchdock web server. PatchDock is a molecular docking algorithm, based on geometry. The ligand and target protein PDB files were submitted to the PatchDock server for docking analysis, using the default value of RMSD cluster 4.0 and the complex form of protein-small ligand as the parameters for analysis. The PatchDock study yielded results for the complementarity score (GSC score) and the geometric interface estimate (AI area)

\subsection{Ligplot analysis using PDBsum:}

LIGPLOT v.4.5.3 PDBsum software was used to predict drug hydroxychloroquine binding sites in the Envelop protein, PLpro protein and Spike glycoprotein receptors. Mechanically, the LIGPLOT software produces 2-D schematic representations of protein-ligand complexes from PDB files. The LIGPLOT diagram displayed the schematic description of all the connections made between the ligand and the residues of protein molecules in the structure (hydrogen bonds and unbonded contacts) [39]; [40].

\section{Results:}

\subsection{Protein structure modeling, validation, energy minimization, and binding site prediction:}


99.89 per cent similarity with PDB ID: 7BTF_A (Chain A, SARS-CoV-2 NSP12 of Severe acute respiratory syndrome coronavirus 2) and Spike glycoprotein showed 99.50 per cent similarity with PDB ID: 6VSB_A (Chain A, SARS-CoV-2 spike glycoprotein of Severe acute respiratory syndrome coronavirus 2). All target protein structure were model using swiss model web server using above PDB structures as template (Fig. 1).

The protein structure validation scores before and after energy minimizations are summarized in Table 1.In ProSA Envelop protein showed minimal while PLPRO protein and spike glycoprotein showed good energy minimization in protein structure. In ModRefiner, all the built structures showed significant changes in RMSD score and result in more stable structure. In Ramachandran plot analysis by Procheck web server, there are significant change in favour amino acid region of Envelop protein, PLPRO protein and spike glycoprotein of SARS-CoV (Table 1).

Table 1: Comparison of all protein structure validation score before and after minimization of target protein from webservers PRoSA,PRocheck

\begin{tabular}{|c|c|c|c|}
\hline Protein targeting & $\begin{array}{l}\text { Protein validation } \\
\text { server }\end{array}$ & Before EM & After EM \\
\hline \multirow{3}{*}{ Envelop protein } & ProSA (Z-score) & -0.87 & -0.59 \\
\hline & Procheck & $84.4 \%$ aa in favorable region & $\begin{array}{c}88.9 \% \text { aa in favorable } \\
\text { region }\end{array}$ \\
\hline & ModRefiner & $\mathrm{RMSD}=5.0$ & RMSD $=0.436$ \\
\hline \multirow{3}{*}{ PLpro protein } & ProSA (Z-score) & -14.18 & -12.87 \\
\hline & Procheck & $89.9 \%$ aa in favorable region & $\begin{array}{l}97.2 \% \text { aa aa in favorable } \\
\text { region }\end{array}$ \\
\hline & ModRefiner & $\mathrm{RMSD}=10.127$ & RMSD=1.056 \\
\hline \multirow{3}{*}{ Spike glycoprotein } & ProSA (Z-score) & -13.79 & -11.57 \\
\hline & Procheck & $84.8 \%$ aa in favorable region & $\begin{array}{c}91.6 \% \text { aa aa in favorable } \\
\text { region }\end{array}$ \\
\hline & ModRefiner & RMSD=11.989 & RMSD $=0.642$ \\
\hline
\end{tabular}
hetero atoms and the ligands are isolated as target proteins. The castp server tells about the cavities and pocket formed by various amino acid in all the target protein structures. The protein structure and predicted active site regions are shown in Fig.2. 
VAL175 in amino acids residues formed cavities and pocket which are play a considerable task in binding and catalytic activity in the active site of PLPRO protein and spike glycoprotein of SARS-CoV2.

\subsection{Ligand Preparation and Evaluation of Lipinski parameters:}

The compound hydroxychloroquine as ligand from pubchem database with id 3652 downloaded in SDF format. Later using Open Babel tool translated in PDF format. Lipinski ligand law review was conducted using Molinspiration with expected properties passed as $\log \mathrm{A}$, Natoms, Mol. Wt., number of donor and acceptor hydrogen bonds, number of rotatable bonds and volume and structure shown in Table 2.

Table 2: Lipinski rule passed by compound hydroxychloroquine

\begin{tabular}{|c|c|c|c|c|c|c|}
\hline Volume & miLog p & TPSA & MW & nON & nOHNH & Volume \\
\hline hydroxychloroquine & 4.0 & 48.38 & 335.88 & 4 & 2 & 321.38 \\
\hline
\end{tabular}

\subsection{Absorption, distribution, metabolism, excretion, and toxicity screening:}

The compound hydroxychloroquine as ligand passed all the ADMET filters in SwissADME. Consensus Log Po/w value is 3.37. Good water solubility with high GI absorption with TPSA 48.39 Å2. The ligands had 0 violation of Lipinski's rules. It also clear the PAIN filter easily (Table 3). The ligand hydroxychloroquine also passed the toxicity prediction by PROTOX-II and admetSAR server. In PROTOX-II, the ligand fall under class-4 with LD50 as $1240 \mathrm{mg} / \mathrm{kg}$ while in admetSAR server, fall under class-3 and does not have any carcinogenic property (Table 4).

Table 3: ADME properties of hydroxychloroquine predicted by SwissADME

\begin{tabular}{|c|c|c|c|c|c|c|c|}
\hline \multirow{2}{*}{ Ligands } & \multicolumn{9}{|c|}{ SwissADME } \\
\hline & $\begin{array}{c}\text { Consensu } \\
\text { s Log } \\
\text { Po/w } \\
\begin{array}{c}\text { hydroxych } \\
\text { loroquine }\end{array}\end{array}$ & $\begin{array}{c}\text { Water } \\
\text { solubility }\end{array}$ & $\begin{array}{c}\text { GI } \\
\text { absorption }\end{array}$ & $\begin{array}{c}\text { Drug- } \\
\text { likeness }\end{array}$ & $\begin{array}{c}\text { TPSA } \\
(\text { \&2) }\end{array}$ & $\begin{array}{c}\text { Lipinski's } \\
\text { rule of five }\end{array}$ & Pain \\
\cline { 2 - 7 } & 3.37 & soluble & High & Yes & 48.39 & 0 Violation & 0 Alert \\
\hline
\end{tabular}

Table 4: Toxicity prediction of hydroxychloroquine predicted by PROTOX-II and admetSAR server

\begin{tabular}{|c|c|c|c|c|}
\hline \multirow{2}{*}{ Ligands } & \multicolumn{2}{|c|}{ PROTOX-II } & \multicolumn{2}{c|}{ admetSAR } \\
\hline \multirow{2}{*}{ hydroxychloroquine } & Class & $\begin{array}{c}\text { LD50 } \\
(\mathbf{m g} / \mathrm{kg})\end{array}$ & Class & Carcinogenicity \\
\cline { 2 - 5 } & 4 & $1240 \mathrm{mg} / \mathrm{kg}$ & III & Non-required \\
\hline
\end{tabular}


The ligand hydroxychloroquine were docked at the binding sites of Envelop protein, PLPRO protein and spike glycoprotein of SARS-CoV2 using Patchdock server, that resulted in energy-based descriptors like energy, intermol energy, torsional energy, internal energy measure of ligand was performed with estimated binding energy $-189.95 \mathrm{kcal} / \mathrm{mol},-216.15 \mathrm{kcal} / \mathrm{mol}$ and $-228.89 \mathrm{kcal} / \mathrm{mol}$ respectively as shown in Fig. 3, in Fig. 4 and Table 5.

Table 5: Docking calculations depicting interacting residues and Binding affinity of target proteins with Ligand

\begin{tabular}{|c|c|c|c|}
\hline ligand & Protein Name & Interacted residues & $\begin{array}{l}\text { Binding affinity } \\
\text { (kcal/mol) }\end{array}$ \\
\hline \multirow{3}{*}{ Hydroxychloroquine } & $\begin{array}{l}\text { Envelop } \\
\text { protein }\end{array}$ & $\begin{array}{c}\text { Phe26,Leu27,Thr30,Leu34, } \\
\text { Ile46,Val47 }\end{array}$ & -189.95 \\
\hline & $\begin{array}{l}\text { PLPRO } \\
\text { protein }\end{array}$ & $\begin{array}{c}\text { Phe833,Ile834,Lys835, } \\
\text { Gln836,Pro862,Pro863, } \\
\text { Thr866,Asp867, }\end{array}$ & -216.15 \\
\hline & $\begin{array}{c}\text { Spike } \\
\text { glycoprotein }\end{array}$ & $\begin{array}{c}\text { Thr319,Leu323,Cys395, } \\
\text { Phe396,Arg456,Tyr457, } \\
\text { Pro460,Pro676 }\end{array}$ & -228.89 \\
\hline
\end{tabular}

193

\subsection{Ligplot analysis using PDBsum:}

In Ligplot analysis, 6 binding residues (Phe26,Leu27,Thr30,Leu34, Ile46,Val47) were predicted in Envelop protein while 8 binding residues (Phe833, Ile834, Lys835, Gln836, Pro862, Pro863, Thr866, Asp867) from PLPRO protein and (Thr319, Leu323, Cys395, Phe396, Arg456, Tyr457, Pro460, Pro676) Spike glycoprotein which were involved in interaction against the ligand hydroxychloroquine as shown in Fig. 5 and Table 5 .

\section{Discussions:}

With millions of people suffering from SARS-CoV2 in Asian and European countries, there is a necessity to find a cure of pandemic COVID-19 as potent drugs which have minimal side effects on administration until vaccination achieved. Protein structure with a resolution of $<2 \mathrm{~A}^{0}$ and identity above $90 \%$ with the query protein sequence serves as a better template for homology modelling of dihydropteroate synthase protein of Mycobacterium leprae [26]. Homology analysis of SARS-CoV2 envelope protein was carried out for identified potential ion channel inhibitor [23]. Homology modelling of SARS-CoV2 receptor binding domain of PLPRO protein used against FDA approved drugs [9]. Ravindra and Kalaria, 2019 [21] also perform the validation of homology structure using Ramachandran plot of Tomato Leaf Curl Virus coat protein. Predicted homology model were subjected to validation using PRoSA based on Z-score and Procheck based on favourable amino acid. The models were further subjected to recheck the above properties after energy minimization to prepare for In silico protein -ligand docking studies [26].Twelve active site were predicted in Cathepsin L in SARS CoV that could be used as potent drug target. Similar 86 anti staphylococcal compounds as ligand were 
eventually passed from by Lipinski using Molinspiration tool and ADMET filters for potent inhibitor against ClfA protein in Staphylococcus aureus using SwissADME tool [40] Lipinski's rule of five helps to determine drug likeness of the compound; an orally active drug should not violate more than the rule. ligand showed 0 violations. Similarly work was carried out for phytochemicals as ligand against dihydropteroate synthase protein of Mycobacterium leprae. The ligands were also passed the toxicity prediction by PROTOX-II with LD50 = $2500 \mathrm{mg} / \mathrm{kg}$ and ligands as non carcinogenic using admetSAR server [40]. Using Ligplot, Thr326, Glu329, Arg602, Arg342, Gly387, Glu388, Val346, Glu337. Arg341, Arg344, Asn330, Lys333 are the active site residues located in receptor-binding domain of Cathepsin L in SARS CoV which are play a considerable task in binding and catalytic activity in the active site of protein. The docking outcome point out that ligand CID11496897 with Cathepsin L in SARS CoV reported $-7.4 \mathrm{kcal} / \mathrm{mol}$ binding energy [29]. The binding affinity of dapsone ligands predicted by AutoDock Vina toward target protein binding site is $-6.7 \mathrm{kcal} / \mathrm{mol}$ [40].

Since the structure and mechanism of action of antimalarial and anti-inflammatory drug (HCQ) ar precisely the same apart from an extra radical moiety in one HCQ terminal, each function a weak base which will alter the hydrogen ion concentration of acidic intracellular organels like endosomes / lysosomes, necessary for membrane fusion. All the agents ar assumed to be powerful instruments against SARSCoV-1 and SARS-CoV-2 [41]; [42]. Associate vital drawback that is still, though, is whether or not HCQ contains a similar impact on SARS-CoV-2 infection. Some information indicate that HCQ effectively repressed all SARS-CoV-2 entry, transport and postentry levels, kind of like antimalarial, and one study found that HCQ was a additional chemical agent than antimalarial in inhibiting SARS-CoV-2 in vitro [43]; [44]. The introduction of radical molecule renders HCQ less pervious to the blood-retinal barrier and permits for faster clearance of retinal pigment cells, indicating a lower risk of HCQ retinal toxicity compared to antimalarial [45]. Additionally, the tiny antimalarial therapeutic and protection index margin makes HCQ an additional stable different than antimalarial.

5. Conclusion:

SARS-CoV-2's history has not been completely elucidated. There is a necessity for designing drugs for SARS-CoV-2 infections caused in human as it has remained an opportunistic pathogen in which causes significant number of the serious and deadly life losses in human. There is actually no COVID-19 pandemic vaccine and contamination is spreading across the globe and there is a compelling need for possible drug management. In the current scenario, we have carried out computational interaction analysis of hydroxychloroquine drugs, which is used as combating the COVID-19 pandemic in worldwide with specific binding sites of Envelop protein, PLPRO protein and spike glycoprotein of SARS-CoV2.

Furthermore, concentrates in vitro and in vivo are required to approve this outcome. Similar in silico docking examination uncovered that hydroxychloroquine drugs dealing with SARS-CoV2 show greater binding affinity with spike glycoprotein and PLPRO protein contrast with envelop protein that could be stepping stool for another aniviral medicate focusing on and creation in future. We expect that these examinations will be steady for structuring a novel and powerful inhibitors against the SARS-CoV2. The minimal effort of chloroquine and HCQ could likewise be a viable technique to battle COVID-19 (particularly in patients with diabetes and other high mortality co-morbidities) in asset obliged and COVID-19 overburdened healthcare 
Conflict of Interest: The authors declare no potential conflicts of interest.

253

254

255

256

257

258

259

260

261

262

263

264

265

266

267

268

269

270

271

272

273

274

275

276

277

278

279

280

281

282

283

\section{Reference}

1. Rota PA, Oberste MS, Monroe SS, Nix WA, Campagnoli R, Icenogle JP, Penaranda S, Bankamp B, Maher K, Chen M-h (2003) Characterization of a novel coronavirus associated with severe acute respiratory syndrome. science 300 (5624):1394-1399

2. Perlman S, Netland J (2009) Coronaviruses post-SARS: update on replication and pathogenesis. Nature reviews microbiology 7 (6):439-450

3. Hui DS, Azhar EI, Madani TA, Ntoumi F, Kock R, Dar O, Ippolito G, Mchugh TD, Memish ZA, Drosten C (2020) The continuing 2019-nCoV epidemic threat of novel coronaviruses to global health-The latest 2019 novel coronavirus outbreak in Wuhan, China. International Journal of Infectious Diseases 91:264

4. Wan Y, Shang J, Graham R, Baric RS, Li F (2020) Receptor recognition by the novel coronavirus from Wuhan: an analysis based on decade-long structural studies of SARS coronavirus. Journal of virology 94 (7)

5. Gorbalenya AE (2020) Severe acute respiratory syndrome-related coronavirus-The species and its viruses, a statement of the Coronavirus Study Group. BioRxiv

6. Woo PC, Huang Y, Lau SK, Yuen K-Y (2010) Coronavirus genomics and bioinformatics analysis. viruses 2 (8):1804-1820

7. Cui J, Li F, Shi Z-L (2019) Origin and evolution of pathogenic coronaviruses. Nature reviews Microbiology 17 (3):181-192

8. Chen Y, Liu Q, Guo D (2020) Emerging coronaviruses: genome structure, replication, and pathogenesis. Journal of medical virology 92 (4):418-423

9. Arya R, Das A, Prashar V, Kumar M (2020) Potential inhibitors against papain-like protease of novel coronavirus (SARS-CoV-2) from FDA approved drugs.

10. Lee S-J, Silverman E, Bargman JM (2011) The role of antimalarial agents in the treatment of SLE and lupus nephritis. Nature Reviews Nephrology 7 (12):718

11. Mizui T, Yamashina S, Tanida I, Takei Y, Ueno T, Sakamoto N, Ikejima K, Kitamura T, Enomoto N, Sakai T (2010) Inhibition of hepatitis C virus replication by chloroquine targeting virus-associated autophagy. Journal of gastroenterology 45 (2):195-203

12. Delvecchio R, Higa LM, Pezzuto P, Valadão AL, Garcez PP, Monteiro FL, Loiola EC, Dias AA, Silva FJ, Aliota MT (2016) Chloroquine, an endocytosis blocking agent, inhibits Zika virus infection in different cell models. Viruses 8 (12):322 
13. Dowall SD, Bosworth A, Watson R, Bewley K, Taylor I, Rayner E, Hunter L, Pearson G, Easterbrook L, Pitman J (2015) Chloroquine inhibited Ebola virus replication in vitro but failed to protect against infection and disease in the in vivo guinea pig model. The Journal of general virology 96 (Pt 12):3484

14. Kouroumalis E, Koskinas J (1986) Treatment of chronic active hepatitis B (CAH B) with chloroquine: a preliminary report. Annals of the Academy of Medicine, Singapore 15 (2):149-152

15. Keyaerts E, Li S, Vijgen L, Rysman E, Verbeeck J, Van Ranst M, Maes P (2009) Antiviral activity of chloroquine against human coronavirus OC43 infection in newborn mice. Antimicrobial agents and chemotherapy 53 (8):3416-3421

16. Blau DM, Holmes KV (2001) Human coronavirus HCoV-229E enters susceptible cells via the endocytic pathway. In: The Nidoviruses. Springer, pp 193-198

17. Kono M, Tatsumi K, Imai AM, Saito K, Kuriyama T, Shirasawa H (2008) Inhibition of human coronavirus 229E infection in human epithelial lung cells (L132) by chloroquine: involvement of p38 MAPK and ERK. Antiviral research 77 (2):150-152

18. Gao J, Tian Z, Yang X (2020) Breakthrough: Chloroquine phosphate has shown apparent efficacy in treatment of COVID-19 associated pneumonia in clinical studies. Bioscience trends

19. Jie Z, He H, Xi H, Zhi Z (2020) Multicenter Collaboration Group of Department of Science and Technology of Guangdong Province and Health Commission of Guangdong Province for Chloroquine in the Treatment of Novel Coronavirus Pneumonia. Expert Consensus on Chloroquine Phosphate for the Treatment of Novel Coronavirus Pneumonia [in Chinese] 10:1001-0939.2020

20. Bhatt MH, Prajapati CK, Reddy M (2017) In silico docking studies of Lupeol with MAPK pathway proteins-Raf-1, MEK \& ERK. Journal of experimental therapeutics \& oncology $12(2)$

21. Ravindra S, Kalaria R (2019) Molecular Identification and in silico Characterization of Coat Protein in Tomato Leaf Curl Virus Associated in Tomato from South Gujarat Region of India. Int J Curr Microbiol App Sci 8 (7):456-466

22. Altschul SF, Gish W, Miller W, Myers EW, Lipman DJ (1990) Basic local alignment search tool. Journal of molecular biology 215 (3):403-410

23. Gupta MK, Vemula S, Donde R, Gouda G, Behera L, Vadde R (2020) In-silico approaches to detect inhibitors of the human severe acute respiratory syndrome coronavirus envelope protein ion channel. Journal of Biomolecular Structure and Dynamics (justaccepted):1-17 
24. Arnold K, Bordoli L, Kopp J, Schwede T (2006) The SWISS-MODEL workspace: a web-based environment for protein structure homology modelling. Bioinformatics 22 (2):195-201 25. Ortega JT, Serrano ML, Pujol FH, Rangel HR (2020) Role of changes in SARS-CoV-2 spike protein in the interaction with the human ACE2 receptor: An in silico analysis. EXCLI journal 19:410

26. Halder ST, Dhorajiwala TM, Samant LR (2019) Multiple docking analysis and In silico absorption, distribution, metabolism, excretion, and toxicity screening of anti-leprosy phytochemicals and dapsone against dihydropteroate synthase of Mycobacterium leprae. International journal of mycobacteriology 8 (3):229

27. Wiederstein M, Sippl MJ (2007) ProSA-web: interactive web service for the recognition of errors in three-dimensional structures of proteins. Nucleic acids research 35 (suppl_2):W407-W410

28. Laskowski RA, MacArthur MW, Moss DS, Thornton JM (1993) PROCHECK: a program to check the stereochemical quality of protein structures. Journal of applied crystallography 26 (2):283-291

29. Saddala M, Kiran J, Rani U (2016) In Silico Drug Design and Molecular Docking Studies of Potent Inhibitors against Cathepsin-L (Ctsl) for Sars Disease. J Res Development 4 (145):2

30. O'Boyle NM, Banck M, James CA, Morley C, Vandermeersch T, Hutchison GR (2011) Open Babel: An open chemical toolbox. Journal of cheminformatics 3 (1):33

31. Borra NK, Kuna Y (2013) Evolution of toxic properties of anti alzheimer's drugs through Lipinski's rule of five. Int J Pure App Biosci 1 (4):28-36

32. Daina A, Michielin O, Zoete V (2017) SwissADME: a free web tool to evaluate pharmacokinetics, drug-likeness and medicinal chemistry friendliness of small molecules. Scientific reports 7:42717

33. Banerjee P, Eckert AO, Schrey AK, Preissner R (2018) ProTox-II: a webserver for the prediction of toxicity of chemicals. Nucleic acids research 46 (W1):W257-W263

34. Yang H, Lou C, Sun L, Li J, Cai Y, Wang Z, Li W, Liu G, Tang Y (2019) admetSAR 2.0: webservice for prediction and optimization of chemical ADMET properties. Bioinformatics 35 (6):1067-1069

35. Cheng F, Li W, Zhou Y, Shen J, Wu Z, Liu G, Lee PW, Tang Y (2012) admetSAR: a comprehensive source and free tool for assessment of chemical ADMET properties. ACS Publications,

36. Baell JB, Holloway GA (2010) New substructure filters for removal of pan assay interference compounds (PAINS) from screening libraries and for their exclusion in bioassays. Journal of medicinal chemistry 53 (7):2719-2740 
37. Yadav S, Pandey SK, Singh VK, Goel Y, Kumar A, Singh SM (2017) Molecular docking studies of 3-bromopyruvate and its derivatives to metabolic regulatory enzymes: Implication in designing of novel anticancer therapeutic strategies. PloS one 12 (5)

38. Schneidman-Duhovny D, Inbar Y, Nussinov R, Wolfson HJ (2005) PatchDock and SymmDock: servers for rigid and symmetric docking. Nucleic acids research 33 (suppl_2):W363W367

39. Wallace AC, Laskowski RA, Thornton JM (1995) LIGPLOT: a program to generate schematic diagrams of protein-ligand interactions. Protein engineering, design and selection 8 (2):127-134

40. Wadapurkar RM, Shilpa M, Katti AKS, Sulochana M (2018) In silico drug design for Staphylococcus aureus and development of host-pathogen interaction network. Informatics in Medicine Unlocked 10:58-70

41. Biot C, Daher W, Chavain N, Fandeur T, Khalife J, Dive D, De Clercq E (2006) Design and synthesis of hydroxyferroquine derivatives with antimalarial and antiviral activities. Journal of medicinal chemistry 49 (9):2845-2849

42. Colson P, Rolain J-M, Lagier J-C, Brouqui P, Raoult D (2020) Chloroquine and hydroxychloroquine as available weapons to fight COVID-19. Int J Antimicrob Agents 105932 (10.1016)

43. Liu J, Cao R, Xu M, Wang X, Zhang H, Hu H, Li Y, Hu Z, Zhong W, Wang M (2020) Hydroxychloroquine, a less toxic derivative of chloroquine, is effective in inhibiting SARS-CoV-2 infection in vitro. Cell discovery 6 (1):1-4

44. Yao X, Ye F, Zhang M, Cui C, Huang B, Niu P, Liu X, Zhao L, Dong E, Song C (2020) In vitro antiviral activity and projection of optimized dosing design of hydroxychloroquine for the treatment of severe acute respiratory syndrome coronavirus 2 (SARS-CoV-2). Clinical Infectious Diseases

45. Marmor M, Kellner U, Lai T, Melles R, Mieler W (2016) American academy of ophthalmology. Recommendations on screening for chloroquine and hydroxychloroquine retinopathy (2016 revision) Ophthalmology 123 (6):1386-1394 